With the Compliments of Springer Publishing Company, LLC

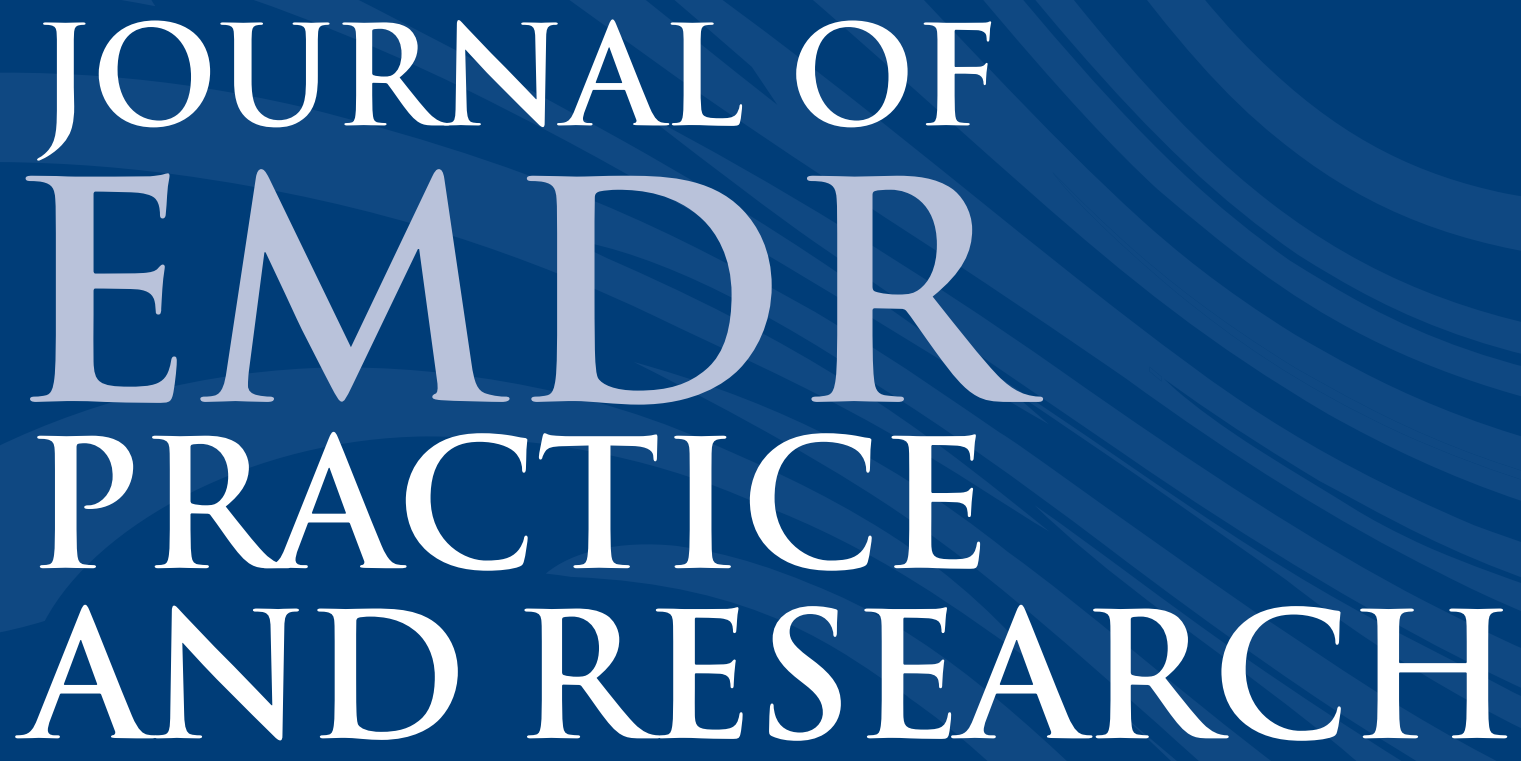

SPRINGER/PUBLISHING COMPANY

www.springerpub.com/emdr 


\title{
ARTICLES
}

\section{Participants' Experiences of EMDR Training in the United Kingdom and Ireland}

\author{
Derek Farrell \\ University of Worcester, United Kingdom \\ Paul Keenan \\ Edge Hill University, Lancashire, United Kingdom
}

\begin{abstract}
This research projects spans a 6-year period surveying 485 participants' experiences of eye movement desensitization and reprocessing (EMDR) training in the United Kingdom and Ireland between the periods of 2005 and 2011. This research used a mixed research methodology exploring EMDR training participants' application of EMDR within their current clinical practice. The rationale was to explore potential differences between EMDR-accredited and EMDR-nonaccredited clinicians in relation to retrospective reports of treatment. Results indicate that EMDR-accredited clinicians report better treatment outcomes. An argument is presented that EMDR has progressed from a convergent technique to a divergent psychotherapeutic approach. Consequently, the research explored whether current EMDR training is "fit for purpose." A comprehensive model for EMDR training is outlined, proposing the importance of developing more EMDR training in academic institutions.
\end{abstract}

Keywords: EMDR; training; academic; outcome; teaching; learning

I $\mathrm{n}$ its 20-year history, EMDR has evolved from a simple technique into a distinct, integrative, client-centered psychotherapy approach whose theoretical underpinning is that of Shapiro's adaptive information processing (AIP) model (Shapiro, 2007, 2012; Shapiro \& Laliotis, 2011; Solomon \& Shapiro, 2008). Internationally, eye movement desensitization and reprocessing (EMDR) is recognized as an empirically supported psychotherapy in the treatment of posttraumatic stress disorder (PTSD; Bisson \& Andrew, 2007; Korn, 2009; Maxfield, 2009; Rothbaum, Astin, \& Marstellar, 2005).

Recent meta-analyses of EMDR justify the therapeutic claims made regarding the effectiveness of EMDR as a treatment of choice in addressing psychological trauma (van den Hout et al., 2011). However, this poses an interesting dilemma to the international EMDR community (Greenwald \& Shapiro, 2010). One argument is that EMDR's strength rests with its effectiveness as a trauma-based intervention and that it is an empirically supported psychotherapy approach. A counter argument draws on the conceptual framework of AIP and posits that EMDR effectively addresses "dysfunctionally stored memory networks" transcending the narrower psychotrauma focus; it argues that EMDR should have a wider clinical application, much broader than that of PTSD.

Shapiro $(1995,2001,2010,2012)$ considers that AIP principles are the foundation for the procedures and protocols that constitute EMDR and further asserts that those AIP principles predict the treatment effects for both traumatic and nontraumatic disturbing memories. Although research evidence with other disorders is still preliminary and tentative, there is emerging practice-based evidence (PBE) relating to the application of EMDR with many mental health conditions other than PTSD. These include depression (Bae, Kim, \& Park, 2008), body dysmorphic disorder (Brown, McGoldrick, \& Buchanan, 1997), emetophobia (de Jongh, 2012), phantom limb pain 
(de Roos et al., 2010), nonpsychotic morbid jealousy (Keenan \& Farrell, 2000), test anxiety (Maxfield, 2007), intellectual disabilities (Mevissen \& de Jongh, 2010); and sex offenders (Ricci, Clayton, \& Shapiro, 2006).

\section{The Development of EMDR}

Since its inception, EMDR has been through six significant stages in its development (Farrell et al., 2011). First was its inception as an original, quite revolutionary technique in the treatment of PTSD. Second, EMDR was subjected to critical review and consideration, some of which was extremely hostile and acerbic. The third stage witnessed the emergence of dismantling studies particularly into EMDR's distinctive bilateral stimulation component characteristic of the modality. The fourth stage witnessed the emergence of more robust randomized control trials responding to criticism about the robustness and reliability of previous studies in support of EMDR. This then lead to a fifth stage where EMDR received greater recognition and support by political acknowledgment when EMDR was adopted in a multitude of national and international guidelines as an effective evidence-based treatment for PTSD. The ongoing sixth stage relates to the current continuation of both evidence-based practice and PBE for using EMDR with mental health conditions other than PTSD (Farrell et al., 2011).

In the development of EMDR, there is support for the argument that it has moved from its initial position of convergence to one of divergence. At its inception, it was projected as a simple technique, which was described as methodologically and theoretically convergent with psychodynamic, behavioral, cognitive behavioral, and integrative approaches (Shapiro, 1995). Currently, it has shifted to a position of divergence and distinctiveness, with assertions that it is a unique psychotherapeutic approach (Shapiro \& Laliotis, 2010; Shapiro \& Solomon, 2010), with an idiosyncratic treatment protocol at its core, and with a theory that guides case conceptualization.

\section{Training in EMDR}

From its early outset, Shapiro $(1989,1995)$ recognized that to obtain academic credibility for EMDR, it was essential to standardize the training for appropriately qualified mental health workers to ensure treatment fidelity, reliability, and validity. To qualify for EMDR training, participants have to be licensed mental health workers with a preexisting psychotherapeutic training background.
The basic structure of EMDR trainings was established in the early 1990s and centered around a Level 1 and Level 2 format, each spread over two 2.5-day training periods, and the general format of EMDR training in the last 20 years has remained relatively unaltered. Currently, the length of EMDR training ranges from 6 to 12 days depending on the training format and context. This is the case even though EMDR has moved from a model of convergence to one of divergence.

\section{EMDR Is a Secondary \\ Psychotherapeutic Training}

The requirement that EMDR clinicians be previously trained as psychotherapists effectively makes EMDR a secondary psychotherapeutic training. This is very different from training in other psychotherapies, such as psychodynamic, humanistic, and cognitive behavioral therapy (CBT). These therapies are typically taught to university students, and the trainings provide core learning in case conceptualization and treatment application. EMDR is taught to therapists who have already been thoroughly instructed in other approaches and who may already have established their own style of treatment.

\section{Training Therapists in Evidence-Based Practice}

The question then arises-what is the best way to disseminate (new) evidence-based practices and to effectively train clinicians so that they adopt and implement the new treatment? Beidas and Kendall (2010) reviewed research on the dissemination of evidence-based practice and recommended a systemscontextual perspective. They noted two essential aspects of integration: (a) how training influences therapists' knowledge and behavior (i.e., the extent to which therapists show adherence, competence, and skill) and (b) how the work environment (i.e., organizational support and clinical population) influences the therapists' adoption of new practices.

The more successful training methods were seen to employ active rather than passive learning approaches (Cross, Matthieu, Cerel, \& Knox, 2007). The inclusion of behavioral role play also improved therapist integration of the new treatment into their existing practice (Wyman et al., 2008). Beidas and Kendall (2010) concluded that the integration of evidencebased practice into clinical practice is related to the quality of the learning experience, training instruction, and learning resources. They also asserted that the systems context was extremely important when considering the implementation of the newly learned 
therapy. They stated that contextual factors such as therapist variables, organizational support, and client variables play a significant part in effective integration of the training into evidence-based practice (Turner $\alpha$ Saunders, 2006).

\section{EMDR Training and Certification}

Currently, the core aspects for EMDR basic training are a training manual, theory-/ practice-driven active teaching and learning experience, behavioral role plays, and the inclusion of clinical supervision as part of the training experience. That these elements are in line with the ideal models described previously suggests that the format may have the potential to optimize the integration and adoption of EMDR into the trainees' clinical practice. However, research is needed to investigate the effectiveness of training models.

Since 1995 within the United Kingdom and Ireland, approximately 9,000 clinicians have been trained in EMDR basic training. At the time of data collection, there were seven EMDR-approved trainers in the United Kingdom and Ireland, but three have since retired, and there are currently four commercial EMDR Europe/EMDR International Association (EMDRIA)approved training providers, all of whom are separate from the national EMDR Association United Kingdom and Ireland. Training was provided in Northern Ireland in 1999 as part of an EMDR Humanitarian Assistance Programs (EMDR-HAP; for further information, see http://www.emdrhap.org/home/index.php). Since the completion of this study, there has been the emergence of a new university EMDR academic training program in the United Kingdom.

The conventional format for EMDR basic training was originally developed by Shapiro (1995) and the EMDR Institute. Approved accreditation authorities within EMDR are EMDRIA or EMDR Europe. Currently, the main structure of approved EMDR basic training consists of a minimum of 20 hours didactic teaching and 20 hours supervised practice from an EMDR-accredited consultant/clinical supervisor. There are, however, slight variations in the duration of EMDR-approved trainings, with some offering more didactive teaching elements ranging from 6 to 14 days. A further variation relates to the EMDR teaching and learning experience and the size of the trainings themselves ranging from large (approximately 75-100 participants) to small (8-12 participants). The EMDR academic U.K. program is a 14-day training; however, this training also contains a formal assessment, which is an examination where EMDR training participants are required to present a clinical case using EMDR highlighting each of the eight phases of the EMDR protocol. This examination is assessed at master's degree level.

Accreditation for EMDR therapists is important because it defines a minimum standard of practice across all EMDR communities. There are three levels of EMDR accreditation: practitioner, consultant, and trainer. The criteria for seeking EMDR accreditation as a practitioner within EMDR Europe (EMDR Association United Kingdom and Ireland, 2012), for example, requires that the individual

- be a licensed mental health worker,

- has completed EMDR basic training conducted by a recognized EMDR-accredited trainer,

- be a member of their national EMDR organization,

- has received a minimum of 20 hours clinical supervision from an EMDR-accredited clinical supervisor/ consultant, in which the EMDR clinical supervisor/ consultant supervising the applicant has directly witnessed the applicants EMDR work either through the use of video/DVD or in vivo, and

- has completed a minimum of 50 sessions and worked with a minimum of 25 clients.

Within the United Kingdom and Ireland, only $10 \%-12 \%$ of trainees who complete EMDR basic training go on to become accredited in EMDR.

\section{The Current Study}

\section{Purpose}

This study evaluated the extent to which EMDR basic trainings result in the integration of the therapy into clinical practice. It also investigated the contextual aspects of integration-therapist variables, organizational factors, and clinical population. In our analysis of the data in this article, we further consider how the implementation of EMDR is being impacted by the expectations that EMDR should be effective for clients with disorders other than PTSD, and we ask whether the current training format for EMDR is adequate for this broader purpose.

This study wished to explore EMDR trainees' experiences of their EMDR training within the United Kingdom and Ireland and to consider the ways in which EMDR was being used, or not, by EMDR trainees back in their clinical working environment. Shapiro (2007) has stated that the goals of therapy are often greatly influenced by the clinicians' original psychotherapeutic paradigm and their personal worldview and, therefore, this research wished to explore some of the attributes of this. A secondary factor regarding the rationale of this study centered 
on the development of a new university-based EMDR training and identifying what needed to be considered in offering a comprehensive, academic, postgraduate training program in EMDR.

For this study, the research posited three questions:

1. Consider how frequently training participants report using EMDR, or not, within their current clinical practice.

2. Explore any differences in retrospective reported treatment outcomes between accredited and nonaccredited EMDR clinicians.

3. To consider, critically, the case for developing EMDR within an academic, postgraduate university training program.

\section{Background to the Research}

This research project was a 6-year (September 2005July 2011) investigation into participants' experiences of EMDR training exploring the implications for future developments in the teaching and learning of EMDR. Participants were recruited from trainings, which took place in Ireland or in the United Kingdom. This was a third stage evaluation postdoctoral research project funded by a research grant awarded by the University of Birmingham, United Kingdom. In addition, the research was a collaborative project with Edge Hill University, Lancashire.

\section{Method}

The primary means of data acquisition was through the use of a postal survey, which asked 19 questions. The structure of the questionnaire used a mixed methodology collecting both quantitative and qualitative material. The entire questionnaire is reproduced in Table 1.

In relation to the clinical use of EMDR, research participants were asked to retrospectively consider their EMDR practice and outcome of treatment. This was a purely subjective measure assessing the research participants' opinion. No standardized measures of treatment outcome or treatment fidelity were employed, so it was not possible to evaluate or triangulate the accuracy of the participants' responses.

\section{TABLE 1. Structure of the Participant Survey of EMDR Training Within United Kingdom and Ireland}

1. What is your core profession?

2. What do you consider to be your main psychological treatment orientation prior to commencing EMDR basic training?

3. Please indicate whether you have completed your EMDR basic training. (Yes/No)

4. Please indicate when you completed your EMDR basic training Level 1.

5. Please indicate when you completed your EMDR basic training Level 2.

6. If you have not completed your EMDR basic training Level 2, please specify your reasons for not doing so.

7. Do you use EMDR as part of your current clinical practice? (Yes/No)

8. If "yes," how often do you use EMDR: daily, weekly, monthly, or rarely?

9. If "no," please provide reason(s) as to why this maybe the case?

10. Since completing your EMDR basic training, how many clients have your treated using EMDR?

11. Please indicate the main type(s) of client issues with which you employ EMDR.

12. Of the clients you have treated to date using EMDR, please indicate the approximate number you have achieved the following: full resolution of treated symptoms, slight reduction, no change, deterioration, client dropped out or did not complete.

13. Please indicate the average number of treatment sessions undertaken to achieve full resolution of your client's symptoms.

14. In your opinion, is this more or less than before you undertook EMDR basic training?

15. Do you have specific clinical supervision for your EMDR practice? (Yes/No)

16. If so, is your clinical supervisor trained in EMDR?

17. Do you participate in any peer support/clinical supervision group?

18. Please outline the ways in which your EMDR basic training meet your needs and expectations and the ways in which it may not have.

19. Please outline your thoughts and suggestions regarding an "ideal model" of EMDR training. What would you like to see included in a comprehensive EMDR training program in the future? 
Ethical approval was sought, and received, through the University of Birmingham. Permission to carry out the research study was also sought, and received, from the EMDR Association United Kingdom and Ireland.

\section{Procedure}

Data were collected from training participants in several stages. These were as follows:

- Stage 1-2005/2006 - Ireland(EMDR-HAP trainings)

- Stage 2-2007/2009—United Kingdom and Ireland (commercial trainings)

- Stage 3-2009/2011-University of Birmingham Academic EMDR training (commenced 2008) $M$ level module (40 credits)

Each of the commercial training providers was contacted with a request that they provide access to their EMDR trainee database. Some of the approved EMDR trainers initially expressed some concern surrounding the amount of time needed to complete the questionnaire. However, permission was eventually granted by each of the trainers, in principle, to proceed with the research. Nevertheless, two of the seven commercial trainers effectively declined to participate in this study by denying access to their EMDR trainee database. Of the EMDR trainers who agreed to participate, this was conditional on the understanding that the research would make no direct comparison between individual trainings. This assurance was given in writing by the research principal investigator and is reflected in the subsequent presentation of the research findings, with all the research participants considered as a single group.

\section{Participants}

Questionnaires were sent out to two distinct groups: EMDR-accredited members of the EMDR Association United Kingdom and Ireland and trainees who had completed their EMDR basic training and had not joined the national EMDR Association. Initial concern surrounding the time needed to complete the questionnaire was allayed following an overall return rate of $45.5 \%$, with most questionnaires returned containing large quantities of data material.

The total number of mailed questionnaires was 1,178 . These included 333 to EMDR-accredited members of the EMDR Association United Kingdom and Ireland and 845 to those mental health clinicians who had undertaken EMDR basic training but had chosen to not join the EMDR national association and therefore were not EMDR Europe accredited. The purpose of this was to highlight potential distinctions between the two groups, EMDR accredited and EMDR nonaccredited.

In total, 530 questionnaires were received, resulting in a $45 \%$ return rate. There were 45 spoils resulting in 485 questionnaires for analysis using SPSS 17 and NVivo 2.0. These spoils were people who had completed the questionnaire but had not completed their EMDR training.

\section{Results}

\section{Respondents' Core Profession}

Table 2 outlines the results from Question 1 of the research questionnaire, which explored the core profession of those participants who undertook EMDR basic training. The most dominant profession undertaking

TABLE 2. Core Profession of EMDR Training Participants $(N=485)$

\begin{tabular}{lccc}
\hline & EMDR Europe Accredited & Nonassociation/Not Accredited & Total \\
\hline Counseling & $40(19.7 \%)$ & $43(15.2 \%)$ & $83(17.1 \%)$ \\
Medicine & $6(2.9 \%)$ & $6(2.1 \%)$ & $12(2.5 \%)$ \\
Mental health Nursing & $17(8.4 \%)$ & $54(19.1 \%)$ & $71(14.6 \%)$ \\
Psychiatry & $10(4.9 \%)$ & $26(9.2 \%)$ & $36(7.4 \%)$ \\
Psychology & $59(29.1 \%)$ & $102(36.2 \%)$ & $161(33.2 \%)$ \\
Psychotherapy & $67(33 \%)$ & $28(9.9 \%)$ & $95(19.6 \%)$ \\
Social work & $3(1.5 \%)$ & $11(3.9 \%)$ & $14(2.9 \%)$ \\
Other & $1(0.5 \%)$ & $12(4.3)$ & $13(2.7 \%)$ \\
Total & $203(41.8 \%)$ & $282(58.2 \%)$ & 485 \\
\hline
\end{tabular}


TABLE 3. Main Psychological Treatment Orientation of EMDR Training Participants

\begin{tabular}{lcrr}
\hline & EMDR Europe Accredited & Nonassociation/Not Accredited & Total \\
\hline Psychodynamic & $26(12.8 \%)$ & $34(12.1 \%)$ & $60(12.4 \%)$ \\
Humanistic & $13(6.4 \%)$ & $24(8.5 \%)$ & $37(7.6 \%)$ \\
Cognitive behavioral therapy (CBT) & $70(34.5 \%)$ & $141(50.0 \%)$ & $211(43.5 \%)$ \\
Integrationist & $68(33.5 \%)$ & $59(20.9 \%)$ & $127(26.2 \%)$ \\
Other & $26(12.8 \%)$ & $24(8.5 \%)$ & $50(10.3 \%)$ \\
Total & 203 & 282 & 485 \\
\hline
\end{tabular}

EMDR training and who then went on to seek EMDR Europe accreditation was that of psychotherapists, predominantly psychotherapists in private practice. The largest professional group not seeking EMDR Europe accreditation was that of chartered psychologists, mainly clinical psychologists who were registered in the United Kingdom by the Health Professionals Council (HPC). In exploring this in more detail, we found that many psychologists (36.2\%) considered that this registration was sufficient to enable them to practice, and that seeking EMDR Europe accreditation was not seen as either essential or important to their current working environment. Those not seeking accreditation predominantly worked for statutory agencies $(82 \%$ public sector), such as the U.K. National Health Service (NHS), government organizations, or the military.

\section{Main Psychological Treatment Orientation}

Survey item number 2 asked respondents about their pretraining psychological treatment orientation. Table 3 demonstrates the largest proportion of clinicians undertaking EMDR training whose predominant psychotherapeutic paradigm was CBT $(42.5 \%)$. Results also highlighted that, after training, only $34.5 \%$ of CBT therapists sought accreditation, compared to $65.5 \%$ of therapists endorsing other orientations.

\section{Reasons for Not Undertaking Level 2/Part 3}

Survey item numbers 3-6 asked when and if respondents had completed their EMDR training, and if they did not complete it, why this was the case. Because most of the EMDR-approved commercial training providers within the United Kingdom and Ireland offer their EMDR training in separate levels or parts, the survey explored how many of the nonaccredited participants actually had not completed the full EMDR basic training. This qualitative data was analyzed, and the themes were ranked in order. Table 4 outlines the ranked order and frequency of the reasons provided by these participants for not completing their EMDR basic training.

\section{TABLE 4. Qualitative Data Themes-Ranked Order and Frequency of Reasons for Not Undertaking Level $2 /$ Part $3(N=45)$}

1. Lack of funding from main sponsor organization (9)

2. Lack of any EMDR (or any) quality supervision provision locally (7)

3. Lack of confidence in applying EMDR theory to clinical practice (6)

4. Difficulty in accessing appropriate clinical populations for using EMDR (5)

5. Current working environment restricts the use of EMDR for other than PTSD (4)

6. Cost of EMDR training is considered too expensive (3)

7. Cost of EMDR Europe consultant/ clinical supervision is too expensive. (3)

8. Lack of individual opportunity/motivation to practice EMDR (3)

9. Dissatisfaction in the quality of the teaching and learning of the EMDR training itself (2)

10. Unforeseen personal circumstances (1)

11. Dissatisfaction/apathy with EMDR as an effective treatment intervention (1)

12. Critical of the current research evidence base for EMDR (1)

Note. PTSD $=$ posttraumatic stress disorder. 


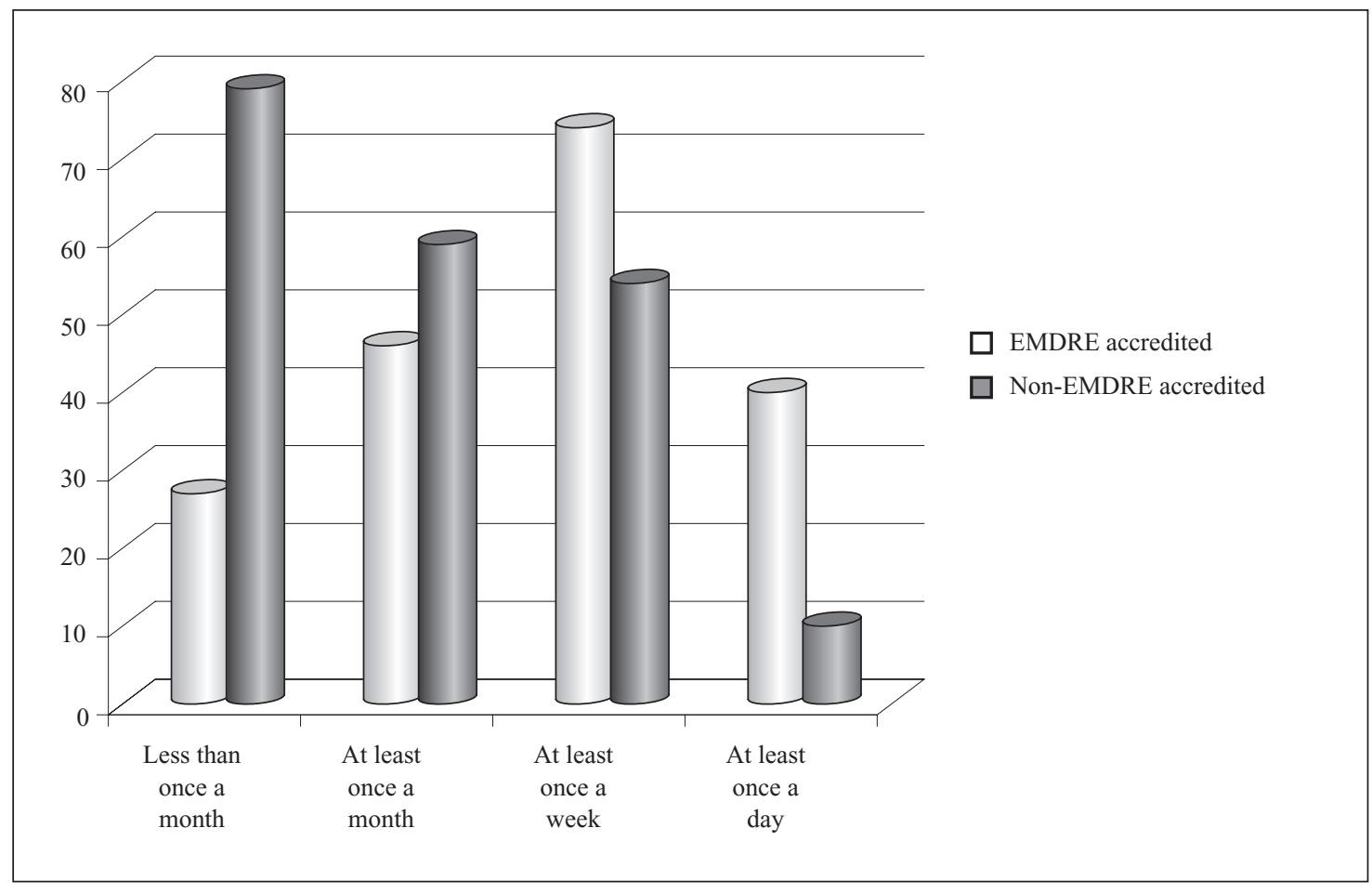

FIGURE 1. Use of eye movement desensitization and reprocessing (EMDR) in current clinical practice $(N=387)$. EMDRE $=$ EMDR Europe.

\section{Current Clinical Use of EMDR}

Survey item numbers 7-10 asked respondents how frequently they used EMDR within their current clinical practice, and if they did not use EMDR, why this was the case. Of the EMDR-accredited members, $91.6 \%$ of participants described themselves as "EMDR clinically active" in comparison to $71.2 \%$ of nonaccredited EMDR clinicians. Figure 1 highlights the frequency with which EMDR-trained clinicians were using EMDR within their current clinical practice. Results indicated a trend that accredited EMDR clinicians seemed more likely to be using EMDR more regularly than nonaccredited participants.

Survey item numbers 11-14 asked about client issues, number of clients treated, and perceived outcome. To further compare the differences between EMDR-accredited and EMDR-nonaccredited clinicians, participants were asked to outline the range of mental health issues with which they used EMDR, and how many clients and how many EMDR sessions they had actually carried out since the completion of their EMDR training. The mental health issues in which clinicians reported using EMDR are outlined in Table 5.

\section{Perceived Outcome}

Survey item number 12 specifically asked about the number of clients treated over the last 12 months with EMDR. Research participants were asked to subjectively rate the outcome of their EMDR intervention with clients, including full resolution, slight resolution, no change, deterioration, and did not complete (DNC) therapy. The responses to this item are reported in Table 6 and were analyzed statistically.

A one-way analysis of variance (ANOVA) was carried out on responses on item number 12 to consider potential differences between the two groups (Hicks, 2004): accredited clinicians (mean $=7.09$, mode $=8$, variance $=3.98$ ) and nonaccredited clinicians (mean $=6.81$, mode $=6$, variance $=3.83$ ). Results indicated that the difference between the two groups was only statistically significant in the full resolution category $(p=.031)$, with the accredited group reporting a larger percentage of full resolution outcomes. There were no significant differences in the other categories of perceived outcomes.

Results also highlighted an interesting distinction between the two groups in the client:clinician ratio. The accreditation group had 47:1 EMDR clients per clinician as opposed to 8:1 for the nonaccreditation group, 
- Single, multiple, ongoing, and complex trauma

- Anxiety disorders including phobias, OCD, and panic disorder

- Traumatic loss, bereavement, and grief

- Depression

- Pain management

- Somatoform disorder

- Body dysmorphic disorder

- Performance enhancement

- Addictions and substance misuse

- Dissociative disorders including DID

- HIV / AIDS

- Oncology

- Recent events and acute trauma

- Psychosis and schizophrenia

Note. $\mathrm{OCD}=$ obsessive-compulsive disorder; DID = dissociative identity disorder.

suggesting a trend that clinicians accredited in EMDR were using EMDR more regularly with clients.

\section{Clinical Supervision for EMDR Practice}

Survey item numbers 15-17 explored issues around EMDR and clinical supervision. Data from the two groups highlighted that a higher percentage of EMDR-accredited clinicians (70\%) than EMDRnonaccredited clinicians $(46.3 \%)$ had a clinical supervision arrangement in place, and that their clinical supervisor was more likely to be trained in EMDR (accredited, 77.7\%; nonaccredited, 49.4\%). Distinctions between both groups are highlighted in Table 7 .

Several participants declared that not only was their supervisor not EMDR trained (accredited, 16\%; nonaccredited, 39.4\%) but also had no expertise either in the field of psychological trauma and that the supervision arranged was in situ purely from a hierarchical and/or organizationally imposed perspective. Results highlighted that 148 clinicians acknowledged practicing EMDR without any clinical supervision at all.

A statistical analysis was conducted to determine if there was a relationship between the reported outcome and the type of supervision received, whether it was provided by an EMDR-accredited consultant or not. Results suggested that supervision by an EMDRaccredited consultant/clinical supervisor was related to outcomes for the accredited therapists $(p=.015)$ but not for the nonaccredited therapists $(p=.093)$.

\section{Evaluation of EMDR Trainings}

Survey item numbers 18-19 asked research participants to evaluate their training experience and to outline their thoughts and suggestions for an ideal

TABLE 6. Total Number (and Percentage) of Clients Treated With EMDR and Reported Outcome

\begin{tabular}{lcrr}
\hline & EMDR Europe Accredited & EMDR Nonaccredited & Total \\
\hline Full resolution & $6,819(72.49 \%)$ & $1,167(52.90 \%)$ & $7,986(68.77 \%)$ \\
Slight resolution & $1,866(19.84 \%)$ & $636(28.83 \%)$ & $2,502(21.54 \%)$ \\
No change & $268(2.85 \%)$ & $170(7.71 \%)$ & $438(3.77 \%)$ \\
Deterioration & $27(0.29 \%)$ & $35(1.59 \%)$ & $62(0.53 \%)$ \\
DNC therapy & $427(4.54 \%)$ & $198(8.98 \%)$ & $625(5.38 \%)$ \\
Total & 9,407 & 2,206 & 11,613 \\
\hline
\end{tabular}

Note. $\mathrm{DNC}=$ did not complete. 


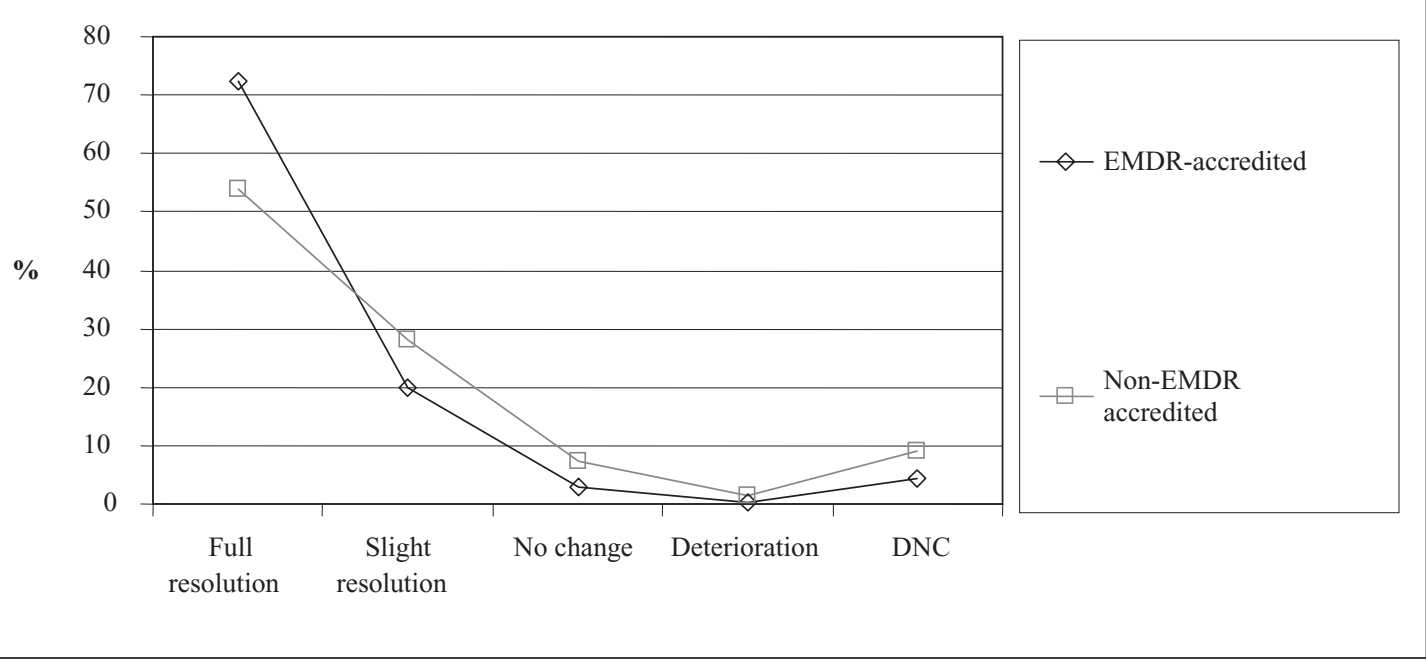

FIGURE 2. Percentage of reported resolution with eye movement desensitization and reprocessing (EMDR) for EMDR-accredited and -nonaccredited EMDR clinicians. DNC = did not complete.

comprehensive training program in EMDR. A qualitative analysis of the data was carried out using NVivo 2.0 to generate the following themes so as to generate core curricula for a comprehensive EMDR training program: EMDR as a psychotherapy approach, EMDR-related essential background and contextual information including core psychometrics, scripted EMDR protocols, EMDR with other clinical populations, more general information, and worksheets when using EMDR. Results are outlined in Figure 3 and discussed in the following text.

\section{Discussion}

\section{Issues Related to Accreditation}

Of the 485 survey respondents, it was interesting to ascertain that the more dominant professional groups to receive EMDR training were primarily psychologists and psychotherapists, with a larger percentage of psychotherapists seeking EMDR accreditation than psychologists. Results may indicate that EMDR clinicians in private practice were potentially more likely to consider the value of seeking accreditation than those employed within statutory agencies (see Table 2). The professional group least likely to seek EMDR accreditation was mental health nurses; this is the group most likely to be employed within the U.K. NHS. Several mental health nurses responded that they considered accreditation in EMDR to gain them "no added value" in terms of their employment or career development. In addition, several participants described case examples where employers asked them to merely be EMDR trained rather than accredited.

This then raises a question: Is EMDR Europe adequately informing trainees about the value of securing EMDR accreditation? Results indicate that the EMDR community may not indeed be currently winning this battle. Several psychologists, particularly those

TABLE 7. Engaged in Clinical Supervision $(N=387)$

\begin{tabular}{lccr}
\hline Clinical Supervision Arrangement & EMDR Europe Accredited & EMDR Nonaccredited & Total \\
\hline Yes & $70.0 \%$ & $46.3 \%$ & $51.73 \%$ \\
No & $26.1 \%$ & $43.91 \%$ & $39.46 \%$ \\
Not answered & $3.9 \%$ & $10.46 \%$ & $8.81 \%$ \\
If yes-supervisor trained in EMDR & $77.7 \%$ & $49.4 \%$ & $56.48 \%$ \\
Supervisor not trained in EMDR & $16.0 \%$ & $39.4 \%$ & $33.55 \%$ \\
Not answered & $6.3 \%$ & $11.2 \%$ & \\
\hline
\end{tabular}




\begin{tabular}{|c|c|c|}
\hline $\begin{array}{l}\text { EMDR as a Psychotherapy } \\
\text { Approach } \\
\text { - } \quad \text { What is EMDR? } \\
\text { - } \quad \text { ThDR in context } \\
\text { processing (AIP) model \& } \\
\text { AIP case conceptualisation } \\
\text { - The three-pronged } \\
\text { protocol (past, present, \& } \\
\text { future) } \\
\text { - The eight phases of EMDR } \\
\text { - Target sequencing plan } \\
\text { Managing strong } \\
\text { emotional responses } \\
\text { Resistance and blocked } \\
\text { processing } \\
\text { Comprehensive } \\
\text { treatment planning vs. } \\
\text { symptom reduction } \\
\text { Appropriate client } \\
\text { selection for EMDR } \\
\text { Complex trauma } \\
\text { populations } \\
\text { Safe/secure place } \\
\text { protocol } \\
\text { Resource installation \& } \\
\text { enhanced safe place } \\
\text { Various methods of } \\
\text { bilateral stimulation } \\
\text { (BLS)/dual attention } \\
\text { stimulus (DAS) } \\
\text { Cognitive interweaves } \\
\text { (process, content, \& } \\
\text { relational) }\end{array}$ & $\begin{array}{cl}\text { Background Information } \\
\text { - } & \text { Psychotraumatology } \\
& \text { Posttraumatic stress } \\
& \text { disorder (PTSD) } \\
\text { - } & \text { Research evidence base } \\
& \text { for PTSD \& EMDR } \\
\text { - } & \text { EMDR \& neuroplasticity } \\
\text { - } & \text { Psychometrics \& EMDR } \\
\text { - } & \text { EMDR research \& } \\
\text { - } & \text { development } \\
& \text { Emerging research in } \\
& \text { support for wider } \\
& \text { applications of EMDR }\end{array}$ & $\begin{array}{cl}\text { Scripted EMDR Protocols } \\
\text { - } & \text { Recent traumatic } \\
& \text { events } \\
\text { - } & \text { Dissociation } \\
\text { - } & \text { Somatic disorder } \\
\text { - } & \text { Phobias } \\
\text { - } & \text { Children \& } \\
& \text { adolescents } \\
\text { - } & \text { Bereavement, loss } \\
& \text { \& grief } \\
\text { - } & \text { Pain } \\
\text { - } & \text { Addictions } \\
\text { - } & \text { EMDR \& self-use } \\
\text { - } & \text { EMDR research } \\
& \text { protocol } \\
\text { - } & \text { OCD } \\
\text { - } & \text { Group child } \\
& \text { protocol } \\
\text { - } & \text { Eating disorders } \\
\text { Phantom limb } \\
\text { - } & \text { pain } \\
& \text { Medical/health- } \\
& \text { related conditions } \\
\end{array}$ \\
\hline
\end{tabular}

FIGURE 3. Proposed outline of a comprehensive training in eye movement desensitization and reprocessing (EMDR). OCD = obsessive-compulsive disorder.

in private practice, emphasized how seeking accreditation over and above being a licensed psychologist seemed unnecessary.

An additional perspective to this argument is conveyed by results (see Table 3), which highlighted that although CBT-trained clinicians compromised the largest group of trainees (43.5\%), they were less likely to seek EMDR accreditation than clinicians with other orientations. This raises some interesting questions. First, why do so many CBT therapists train in EMDR? Considering the relative dominance of CBT in the U.K. health care market place and the strong empirical support for trauma-focused CBT as a treatment intervention for PTSD (National Institute for Health and Clinical Excellence [NICE], 2005), why do they seek further training in trauma therapy? Are they committed to learning and expanding skills, or do they consider themselves insufficiently experienced or skilled to work specifically with trauma (PTSD) populations? Further research could explore motivation for training in EMDR among this group to optimize their experience and learning. Second, why do CBT therapists not seek accreditation? That the results highlight that $50 \%$ of all participants not seeking accreditation were CBT therapists is an area of concern for the EMDR community. 


\section{Completion of Training Programs}

Most of the EMDR-approved commercial training providers within the United Kingdom and Ireland offer their EMDR training with distinct sections. The survey found that just fewer than $10 \%$ did not complete the full EMDR basic training and were of course not able to seek accreditation. Statements provided by the respondents suggested that some quickly discontinued using EMDR after Level 1 training. The reasons for not completing EMDR training were very diverse (see Table 4). However, three main aspects merit further investigation and consideration; these are (a) lack of opportunities for funding, (b) lack of EMDR clinical supervision provision, and (c) lack of confidence in using EMDR.

\section{Reasons Provided for Not Using EMDR}

Several explanations were offered by the 98 participants not using EMDR in their current clinical practice. A third (33\%) considered that the EMDR training they received was insufficient in enabling them to feel suitably equipped and confident in using EMDR. This again raises further questions as to how best the EMDR community can respond to this. Interestingly, $6 \%$ of the participants who are not clinically active stated that the reason they were not using EMDR was because of organizational or institutional bullying. Although a relatively small number of participants, nonetheless, this is still a concerning phenomenon warranting further investigation and research by the EMDR community.

\section{The Cost of Training and Clinical Supervision}

As noted previously, the high cost of training programs and the lack of funding opportunities was noted by some participants as the reason that they were unable to complete their EMDR training. Other respondents stated that the high cost of supervision was the reason that they were unable to achieve accreditation. They described being discouraged from continuing with EMDR accreditation after receiving quotes from some EMDR consultants, charging $£ 150$ an hour for clinical supervision. In market-driven environments, it would be extremely difficult for EMDR national associations to legislate a cap in fees for EMDR clinical supervision. However, we recommend that associations make a commitment to widening the availability of good quality EMDR Europe clinical supervisors or perhaps allowing more of the supervision to be conducted in group settings to help reduce costs for EMDR supervisees. There is an argument that the high costs for EMDR training and EMDR clinical supervision has the potential to discourage EMDR development.

\section{Clinical Applications of EMDR}

Results indicated by Table 5 are, by themselves, not surprising. Nonetheless, the results are an indication of a pressing challenge to the EMDR community. The NICE in the United Kingdom currently only stipulate the use of EMDR for PTSD and perinatal PTSD (NICE, 2005; NICE, 2007a). None of the other NICE clinical guidelines endorses the use of EMDR for anything other than PTSD. This is consistent with the current research evidence; there is only preliminary evidence for the use of EMDR with any other disorder (Maxfield, 2009).

In comparison, CBT is endorsed by NICE, based on current empirical evidence, for the use of schizophrenia (2002); self-harm (2004); anorexia (2004); bulimia (2004); atypical eating disorders (2004); obsessivecompulsive disorder (OCD; 2005); body dysmorphic disorder (2005); PTSD (2005); dementia (2006); depression (2007); anxiety, panic, and generalized anxiety disorder (2007); and perinatal PTSD (2007).

The need for quality research and publication related to the wider applications of EMDR other than for PTSD populations continues to be a much needed area of priority. The fact that so many clinicians train in EMDR and then used it with reported effectiveness with various different populations is to be applauded; however, this needs to be backed up by hard research evidence in support of this endeavor.

There are intriguing questions to consider here. Some of the mental health conditions indicated in Table 5 have less of a traumatological basis than others. It could be argued that the EMDR community supports, although tacitly, the use of EMDR with various disorders (e.g., OCD), even though it is known that the empirical evidence for its use with such disorders is presently fairly weak. This raises questions about accountability, informed consent, and responsibility. There are related issues for EMDR training provision.

Table 5 indicates that EMDR is being used with a wide range of mental health problems. Nevertheless, the treatment of most of these clinical populations is not covered in the EMDR basic trainings because there is little/no research support for these applications. On the other hand, clinicians are daily using EMDR with the various clinical issues, which present in their caseloads. This raises the question as to whether EMDR basic training is sufficient in its teaching and learning 
content. When we examine the data more closely, we note that the current training format appears to have been sufficient in empowering clinicians to use EMDR with broader clinical populations because the respondents reported treating a large clinical population $(11,613$ patients). This is a strong argument. However, an alternative view is that there are several clinicians trained in EMDR who are not using EMDR and who report that the training was not sufficient for their needs and others who use EMDR but with mixed or limited success. Could these challenges be related to the challenges of complex presentations? How should these issues be addressed in training programs?

Another related question is how trainers should address EMDR application with potentially difficult and complex populations, which require specialist knowledge and expertise (e.g., psychosis, pain management, OCD). We recommend discussion by national associations about clinical governance as well as further research to explore this in more detail.

\section{Reported Treatment Outcome}

The data provided by participants about their use of EMDR within their current clinical practice was very revealing. These results must be scrutinized with some degree of caution because they are retrospectively based on purely subjective data provided by the research participants. Consequently, there was no means to independently verify or triangulate the data provided by the research participants.

This study sought to investigate if there were any differences between the reported EMDR treatment outcomes of accredited and nonaccredited therapists. As described previously, an ANOVA was carried out to consider potential differences between these two groups. Figure 2 highlights the statistically significant difference $(p=.038)$ with the accreditation group reporting a significantly higher number of full resolution outcomes only. Although these results indicate that accredited therapists report better outcomes, it is not possible to determine if they actually achieved better outcomes because the data was retrospective and no objective measurements of outcome were administered. There are several possible reasons for the difference in retrospective reported outcomes. First, EMDR-accredited clinicians may potentially be more experienced, or feel more confident, or evaluate the patient's response in a more positive light. The actual correspondence between the therapists' reports and the patient outcome needs further exploration and research. A further explanation may relate to EMDR treatment fidelity. On the assumption that better treatment fidelity produces better outcomes (Maxfield \& Hyer, 2002), are EMDR-accredited clinicians more faithful to EMDR? This also needs further investigation.

An additional argument may be that EMDRaccredited clinicians are potentially better supported, have potentially better clinical supervision, or may even have better systems of consultation in situ as opposed to nonaccreditedEMDR clinicians. Again, this needs further review. In addition, EMDR-accredited clinicians may be more effective in selecting potentially appropriate clients for EMDR. However, further research and investigation is required to explore all these ideas in more detail.

It should be highlighted, however, that nonaccredited EMDR clinicians still get very effective treatment outcomes with EMDR. Nonetheless, the question remains about what extra ingredient accreditation adds treatment outcome.

Another area for potential research centers on the small proportion of clients that research participants considered deteriorated $(0.53 \%)$. It would be interesting to consider whether this was related specifically to EMDR or to significant other factors.

\section{Clinical Supervision}

Our research team found analyzing the data about clinical supervision to be challenging on several levels. There were 387 EMDR clinicians who answered the item about clinical supervision. As Table 7 indicates, only $51.73 \%$ of clinicians were in receipt of clinical supervision and of this percentage, $33.55 \%$ declared that their supervisor was not actually trained in EMDR. Several participants declared that their supervision was provided purely from a hierarchical and/or organizationally imposed perspective, and that their supervisor was not EMDR trained and with no expertise in the field of psychological trauma. Furthermore, 148 clinicians (39.46\%) acknowledged treating 4,456 clients with EMDR without any clinical supervision or support. Although these clinicians are not practicing unprofessionally, nonetheless, it does raise a further question for EMDR national associations regarding clinical governance and EMDR good practice. However, there are several other questions to consider about EMDR clinical supervision:

1. Does the provision of EMDR without supervision raise any concerns for the EMDR community?

2. Is completion of training sufficient for competent treatment practice?

3. Is accreditation essential to establish competency?

4. Once accreditation is completed, does supervision need to continue? 
TABLE 8. Impact of Clinical Supervision

\begin{tabular}{lccc}
\hline Participant Group & $d f$ & $F$ & $p$ \\
\hline EMDR Europe accredited & 1 & 4.37 & $.015^{\star}$ \\
EMDR nonaccredited & 1 & 3.006 & .093 \\
\hline
\end{tabular}

${ }^{\star}$ Significant $p<0.5$.

Licensing boards allow most clinicians to ethically practice psychotherapy independent of clinical supervision once they have completed training. Other clinicians require evidence of clinical supervision as a mandatory part of any reaccreditation criteria. In the United Kingdom, for example, to be reaccredited as a CBT therapist requires confirmation from that clinician's CBT clinical supervisor declaring that the CBT therapist is in receipt of regular CBT clinical supervision. The same is also true for EMDR-accredited practitioners, consultants, and trainers. However, this only applies to those accredited and not to those not accredited.

Table 8 explored the clinical supervision arrangements between accredited and nonaccredited EMDR clinicians. Results seem to suggest an interesting observation that to be EMDR accredited and supervised by an EMDR-accredited clinical supervisor is better than (a) not being accredited, (b) if the supervisor is not trained in EMDR, and (c) having no clinical supervision. However, because these results are based on both subjective and retrospective data, caution must be exercised.

\section{Considerations About EMDR Trainings}

The results from item numbers 18 and 19 of the survey produced copious amounts of material, which then had to be reviewed and analyzed. These were then condensed and contained within Figure 3 in the results section. In considering the content of Figure 3 more closely, there are several questions that should be considered:

1. Are all these subjects relevant to EMDR basic training?

2. Can this amount of content be covered within the present 7-day format?

3. How can we assess both knowledge acquisition and competency without effective means of assessment being built into the EMDR training provision?

4. How can we further promote the clinical credibility of EMDR in recounting its therapeutic effectiveness and its powerfulness as a psychotherapeutic intervention on one hand and the shortness of training on the other?
Figure 3 suggests that the current EMDR basic training format is not meeting training participants' needs in terms of training content. The second point would also highlight the current disconnect between the EMDR training provision and EMDR research and development. To take a different perspective, consider another question: Why is CBT so dominant? One suggestion might be twofold; first, CBT is predominantly taught in academic institutions, and EMDR is not. Currently in the United Kingdom and Ireland, there are 20 academic institutions offering training in CBT; for EMDR, there is one. A second suggestion is that immersion of trainings in academic institutions raises both academic credibility and essentially generates a necessary research culture to justify its placement in academic institutions in the first place. CBT is predominantly taught at master's degree level and above, which has a prerequisite research component as a fundamental part of the academic structure of the program. For CBT, training and research go hand in hand. This is not the same for current EMDR training. It seems important to consider that for EMDR to continue to grow and develop, then EMDR training and research need to go hand in hand. Are academic institutions therefore the best environment to enable this process to happen?

According to Beidas and Kendall (2010), the current "gold standard" in evidence-based practice relating to psychological treatments includes three essential components: (a) a training workshop, (b) a training manual, and (c) clinical supervision. The effectiveness of trainings is not solely based on the content of that training. The way in which people are trained is just as important as the content of a training program. An argument presented by Cross et al. (2007) purported that a training method involving an active learning approach stands a much stronger chance of being better employed within a clinical context. Cross et al. outlined five important elements of active learning: appropriate modeling, opportunities to practice, building self-efficacy, interaction among learners, and behavioral role play. There is an argument to support the view that currently, each of these five elements is part of current EMDR basic training.

As mentioned earlier, since 1995 within the United Kingdom, approximately 9,000 clinicians have been trained in EMDR. Currently, the membership of EMDR United Kingdom and Ireland national association consists of just more than 1,000 members, with the natural conclusion here being that many more people do not join the association than those who do and that following EMDR training, potentially, more are not clinically active with EMDR than those 
who are. So why might this be the case? A further dimension is that less than half of the current EMDR United Kingdom and Ireland national association membership is accredited.

One wonders why this is the case. Does this represent a problem with the training itself? What then might this say about the "fit for purpose" of the current EMDR basic training model? Is merely making EMDR basic training more comprehensive the answer? Or, in light of Beidas and Kendall's (2010) observations, should consideration be given to ensuring that if EMDR is to be better integrated into clinical practice, more attention needs to be given to the provision of robust and effective EMDR clinical supervision.

From this survey, it seems that there are six questions that need further investigation and research:

1. Is the current format of EMDR basic training sufficient for optimal integration into clinical practice?

2. What should be the optimal duration of EMDR basic training?

3. What should be the optimal focus of EMDR basic training?

4. How much of EMDR basic training is based on evidence-based practice?

5. How much EMDR clinical supervision is needed to achieve behavioral change and effective integration into clinical practice?

6. What is the most effective and robust means of assessing competency in EMDR?

\section{Conclusion}

This article has embarked on a critical exploration of the current EMDR training provision and the experiences of participants undertaking EMDR training between 2005 and 2011 in relation to three specific research questions: (a) consider how the training participants were using EMDR, or not, within their current clinical practice; (b) explore any differences between accredited and nonaccredited EMDR clinicians; and (c) to consider, critically, the case for developing EMDR within an academic, postgraduate university training program.

As a mixed methodology that focuses on retrospective evaluation, some caution in the results relating to outcome should be considered. Although the data suggest the potential value of seeking accreditation within EMDR and supervision by an EMDR-accredited consultant/clinical supervisor, this study did not investigate causality, and no determination can be made that accreditation and supervision produce better outcomes. The reason that EMDR-accredited therapists retrospectively report better outcomes was also not investigated, and no conclusions can be drawn about this observation. Further research is needed to explore this potential phenomenon in more detail.

The research findings raise interesting questions about participants' experiences of EMDR training and provide a unique insight into their "ideal" active learning model of an EMDR training syllabus for the future. Considering the potential link between promoting academic EMDR trainings, developing future research and development in EMDR, and integrating EMDR into more broad mental health training programs is an endeavor worthy of further exploration by the EMDR community.

EMDR clinical supervision is crucial for skillful EMDR treatment delivery. Understanding the importance of effective and robust EMDR clinical supervision, its key features, and the contribution it makes toward EMDR basic training driven by evidence-based practice are valuable areas for future research and investigation. Despite the importance of the concept of evidence-based practice, more research is needed to ascertain how best to train clinicians in evidence-based EMDR practice, implementation, and integration into clinical practice.

\section{References}

Bae, H., Kim, D., \& Park, Y. C. (2008). Eye movement desensitization and reprocessing for adolescent depression. Psychiatry Investigation, 5(1), 60-65.

Beidas, R. S., \& Kendall, P. C. (2010). Training therapists in evidence based practice: A critical review of studies from a systems-contextual perspective. Clinical Psychology: Science and Practice, 17, 1-30.

Bisson, J., \& Andrew, M. (2007). Psychological treatment of post-traumatic stress disorder (PTSD). Cochrane Database of Systematic Reviews, (3), CD0003388.

Brown, K. W., McGoldrick, T., \& Buchanan, R. (1997). Body dysmorphic disorder: Seven cases treated with eye movement desensitization and reprocessing. Behavioural and Cognitive Psychotherapy, 25, 203-207.

Cross, W., Matthieu, M., Cerel,J., \& Knox, K. (2007). Proximate outcomes of gatekeeper training for suicide prevention in the workplace: A suicide prevention in the workplace. Suicide and Life-Threatening Behaviour, 37, 659-670.

de Jongh, A. (2012). Treatment of a woman with emetophobia: A trauma focused approach. Mental Illness, 4, e3.

de Roos, C., Veenstra, A. C., de Jongh, A., den HollanderGijsman, M. E., van der Wee, N. J., Zitman, F. G., \& van Rood, Y. R. (2010). Treatment of chronic phantom limb pain using a trauma-focused psychological approach. Pain Research \& Management: The Journal of the Canadian Pain Society, 15(2), 65-71.

Farrell, D. P., Keenan, P. S., Wajid Ali, M., Bilal, S., Tareen, S. M., Keenan, L., \& Rana, M. H. (2011). Training Pakistani mental health workers in EMDR in the aftermath of the 2005 earthquake in Northern Pakistan. Counselling Psychology Quarterly, 24(2) 127-137. 
Greenwald, R., \& Shapiro, F. (2010). What is EMDR?: Commentary by Greenwald and invited response by Shapiro. Journal of EMDR Practice and Research, 4(4), 170-179.

Hicks, C. M. (2004). Research methods for clinical therapists: Applied project design and analysis. New York, NY: Churchill Livingstone.

Keenan, P. S., \& Farrell, D. P. (2000). Treating nonpsychotic morbid jealousy with EMDR, utilising cognitive interweave: A case report. Counselling Psychology Quarterly, 13(2), 175-189.

Korn, D. L. (2009). EMDR and the treatment of complex PTSD: A review. Journal of EMDR Practice and Research, 3(4), 264-278.

Maxfield, L. (2007). Current status and future directions for EMDR research. Journal of EMDR Practice and Research, 1(1), 6-14.

Maxfield, L. (2009). Looking back, moving forward. Journal of EMDR Practice and Research, 3, 210.

Maxfield, L., \& Hyer, L. (2002). The relationship between efficacy and methodology in studies investigating EMDR treatment of PTSD. Journal of Clinical Psychology, 58(1), 23-41.

Mevissen, L., \& de Jongh, A. (2010). PTSD and its treatment in people with intellectual disabilities: A review of the literature. Clinical Psychology Review, 30, 308-316.

National Institute for Health and Clinical Excellence. (2005). Post-traumatic stress disorder (PTSD): The management of adults and children in primary and secondary care. London, United Kingdom: NICE Guidelines.

National Institute for Health and Clinical Excellence. (2007a). Antenatal and postnatal mental health. Clinical Guideline (CG45). London, United Kingdom: NICE Guidelines.

National Institute for Health and Clinical Excellence. (2007b). Schizophrenia (CG82). London, United Kingdom: NICE Guidelines.

Ricci, R. J., Clayton, C. A., \& Shapiro, F. (2006). Some effects of EMDR treatment with previously abused child molesters: Theoretical reviews and preliminary findings. Journal of Forensic Psychiatry and Psychology, 17, 538-562.

Rothbaum, B. O., Astin, M. C., \& Marsteller, F. (2005). Prolonged exposure versus eye movement desensitization and reprocessing (EMDR) for PTSD rape victims. Journal of Traumatic Stress, 18(6), 607-616.
Shapiro, F. (1989). Efficacy of the eye movement desensitization procedure in the treatment of traumatic memories. Journal of Traumatic Stress, 2, 199-223.

Shapiro, F. (1995). Eye movement desensitization and reprocessing, basic principles, protocols and procedures. New York, NY: Guildford Press.

Shapiro, F. (2001). Eye movement desensitization and reprocessing, basic principles, protocols and procedures (2nd ed.). New York, NY: Guildford Press.

Shapiro, F. (2007). Handbook of EMDR and family therapy processes. New York, NY: Wiley.

Shapiro, F. (2010). The human mind, psychotherapy and EMDR. Actas Españolas de Psiquitaria, 38(Suppl. 3), 44-46.

Shapiro, F. (2012). Getting past your past: Take control of your life with self-help techniques from EMDR therapy. New York, NY: Rodale.

Shapiro, F., \& Laliotis, D. (2011). EMDR and the adaptive information processing model: Integrative treatment and case conceptualization. Clinical Social Work Journal, 39(2), 191-200.

Shapiro, F., \& Solomon, R. (2010). Eye movement desensitization and reprocessing. In I. Weiner \& W. E. Craighead (Eds.), The Corsini encyclopedia of psychology (4th ed., Vol. 2). Hoboken, NJ: Wiley. http//:dx.doi,org/ 10.1002/9780470479216.corpsy0337

Solomon, R. W., \& Shapiro, F. (2008). EMDR and the adaptive information processing model: Potential mechanism of change. Journal of EMDR Practice \& Research, 2, 315-325.

Turner, K., \& Sanders, M. (2006). Dissemination of evidencebased parenting and family support strategies: Learning from the Triple P-Positive Parenting Program system approach. Aggression and Violent Behavior, 11, 176-193.

van den Hout, M. A., Engelhard, I. M., Rijkeboer, M. M., Koekebakker, J., Hornsveld, H., Leer, A., . . . Akse, N. (2011). EMDR: Eye movements superior to beeps in taxing working memory and reducing vividness or recollection. Behaviour Research and Therapy, 49, 92-98.

Wyman, P., Brown, C., Inmann, J., Cross, W., SchmeelkCone, K., \& Guo, J. (2008). Randomized trial of a gatekeeper program for suicide prevention: 1-year impact on secondary school staff. Journal of Consulting and Clinical Psychology, 76, 104-115.

Correspondence regarding this article should be directed to Dr. Derek Farrell, University of Worcester, Institute of Health Sciences, United Kingdom. E-mail: D.Farrell@worc.ac.uk 